\title{
Hypovigilant System: An Approach for Lethargy Detection
}

\author{
Revati Bhor \\ SPPU, Pune
}

\author{
Pranjal Mahajan \\ SPPU, Pune
}

\author{
Omkar Dharmadhikari \\ SPPU, Pune
}

\author{
H.V. Kumbhar \\ SPPU, Pune
}

\begin{abstract}
There is much attentional impairment while driving that affect driver's reaction. Among which driving while lethargic is one of the major causes behind road accidents, and exposes the driver to a large extent of crash risk compared to driving while alert. Therefore, the use of an assistive system that monitor a driver's level of vigilance and alert the driver in case of lethargy can be significant in the prevention of accidents. This paper introduces a new approach towards detection of driver's lethargy based on yawning measurement and head movement. This involves several steps which includes the real time detection and tracking of head movement, the detection of yawning based on measuring the amount of changes in the mouth contour area. Test results express that the proposed system is able to measure the aforesaid parameters and detect driver's lethargy.
\end{abstract}

\section{Keywords}

Lethargy detection, Yawn detection, Face detection, Head movement detection

\section{INTRODUCTION}

Lethargy of a driver contributes factor 22 to 24 percent of car crashes according to the National Highway Traffic Safety Administration (NHTSA). Also the accidents caused due to lethargy is four to six times higher than that of near-crash or crash risk relative to alert drivers [1] as fatigue drivers fail to take immediate corrective actions earlier to a collision. Road Safety Ministry of Canada's Driver Handbook also mentions that "Lethargy is a major fact of growing number of collisions resulting in injury and fatal accident. The driver fatigue affects his/her alertness which increases chances of car accidents. Hence drivers tend to make errors which can cause severe damage. Even before driver himself understand that he/she is lethargic, it can influence his ability. As the issue is well studied, it is stated that accidents may cause during late night/early dawn hours (between 2:00 a.m. and 6:00 a.m.) or late afternoon (between 2:00 p.m. and 4:00 p.m.) because of lethargy. The shift workers, people with undiagnosed or untreated slumber disorders, and commercial vehicle operators, are at higher risk for such accidents." An important paradox of driver's lethargy is that the driver may be too tired to become conscious of his own level of distraction. Hence, driver assistance systems which can identify lack of attentiveness and aware the driver of his lethargy will play an essential role in the automation system of future vehicles to avoid accidents and save lives.

Different signs and body gestures can be used as indicators of driver fatigue. They include inattentiveness while on the road, driving over the center line, gaping, feeling impatient, heavy eyes and leisurely reaction. A number of related works have used changes in driver's face (i.e. eye motion, gaping etc.). This paper focuses on yawning and head movement as a sign of fatigue. Unlike existing systems that suffer from computational intricacy, facial hindrance factors, and adverse lighting conditions, this system eliminates these shortcomings.

In this approach, the driver's face is constantly captured using a video camera which is installed on a dashboard in front of driver's face inside the car. Next, detecting lethargy involves three main steps to measure changes in facial gestures that imply fatigue. First, the driver's face is detected and tracked in the series of frame shots taken by the camera. Second, after detection of the face, head movement is detected. Third, the yawning state is detected. Figure [1] demonstrates several steps of the algorithm.

\section{RELATED WORK}

As driver's lethargy is one of the major causes of road accidents, it is well studied and addressed in much research work. This helps to increase transportation safety and decrease the number of deaths caused by fatigued drivers. Different body gestures of driver were used to measure lethargy for which the methods for assessing measurement of the driver's state, driver's performance and a combination of the driver's state and performance were analyzed. Primarily techniques focusing on computer vision were used [2]. Tuncer et al. proposed an assistant system to track a lane for those drivers who were not able to perform a good job of lane keeping [3]. This method was based on calculating steering actions and further those actions were sent to vehicle model. Driver's assessment was determined in the context of a road departure warning and intervention system [4]. The position of vehicle was used as input and the changes in the bandwidth or parameters of such a model were analyzed. Fatigue detection method based on brain and visual activity was proposed by [5]. Another method assessed fatigue by using electrocardiography, eye movement [6]. A pulse wave sensor was used to detect lethargy [7]. Hayashi et al. proposed method of driver's lethargy detection by pulse wave analysis with a neural network [8]. A clustered background based on segmentation was used by Sirohey in order to identify the face [9] as shown in Figure 2. [10] developed a new method based on locating facial gestures. A method of face detection based on a morphology technique to perform eye analogue segmentation was proposed by [11]. [12] introduced a driver's lethargy monitoring system which had used a combination of eyes and mouth gestures. The anticipated structure determines the status of mouth and eyes. The feature points using Back Propagation neural networks were analyzed in order to check for conditions that involve driver drowsiness. [13] used a face detection system that uses histogram intersection in the HSV color space to highlight the skin region. [14] proposed the following method: the frontal view face is detected based on template matching. The extracted edges from sobel filtering were grouped together to locate the face. Then, the same procedure was repeated to find other facial features such as eyes, mouth and nose in the candidate face. Face detection proposing BP neural network and Bayesian decision was described. 

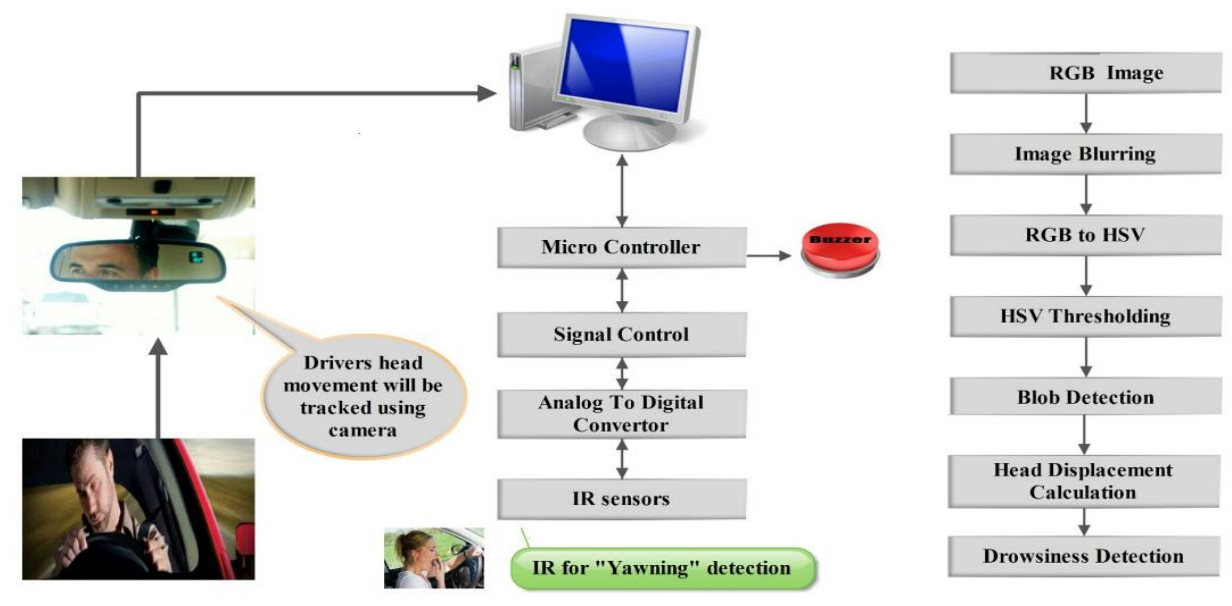

\section{Figure [1] System Architecture}

by Liu et al [15]. The Bayesian decision theory was used to classify the face or non-face pattern and also to improve the correct rate of face detection. A linear arrangement of the facial appearance to detect the yawning mouth was main focus of [16] who took benefit of grey protuberance and Gabor wavelets to distinguish the mouth corners and used LDA. [17] proposed a system to detect the face using ViolaJones technique and extracted the mouth region, in which lips were searched in the course of spatial fuzzy c-means (s-FCM) clustering. [18] exploited mouth geometrical features to detect yawning. [19] used the cascade of classifier which is proposed by Viola-Jones face recognition approach. It then used a support vector machine to train the classifier with the mouth features in yawning condition. Adaboost and multinomial ridge regression were used to prepare the classifier of different facial measures such as blinking and yawn motions [20]. [21] proposed a method of hypo-vigilance detection by processing of the eye region and without an explicit eye detection stage. A lethargy detection method based on eyelid movement was proposed by [22]. [23] used a method of fatigue detection by applying the Structural Similarity Measure to find the eye location. The method to eliminate the frame region in the face area to reduce the search area was described by [24]. [25] required the use of two cameras : a low resolution camera for the face tracking and a high resolution to measure mouth features. It used haar-like features to distinguish driver's mouth, and yawning is detected based on the characteristic ratio of the mouth. Two cameras were used in the proposed method of Li et al.

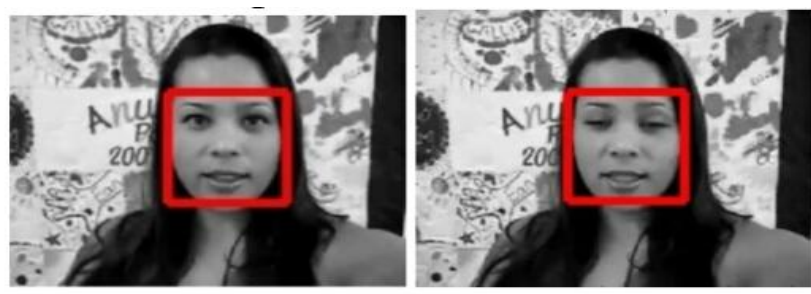

Figure 2

[26] detected driver's fatigue in real time. In this method, one low resolution camera was installed in the car to supply the driver's head position and one high resolution camera to locate the mouth region in each frame. In the method studied by Fan et al. [27], the driver's face was detected by using a Gravity-Center template. Jimenez et al. [28] described a method of fatigue detection based on the percentage of closing eyes and detection of yawning and nodding. The goal of face detection is to categorize all image regions that restrain a face in spite of of its position, orientation, and lighting conditions. Human faces are non-rigid and varies in size, shape, color, and texture. The orientation of the face is also a challenge in the detection process. The other important factor is lighting conditions. The environment light can change depending on the time of the day and weather conditions which affects lighting conditions. [29] addressed the difficulty of face detection in the presence of lighting variations and proposed a system based on the use of an adaptive illumination normalization procedure. In appearance based methods, the face models are learned from a set of training images, which include the representative variability of facial appearance. Such methods can take advantage of Neural Networks. However, when it comes to a yawning face, especially when the mouth is wide open it sometime fails to distinguish the whole face along the bottom of the chin. The presence of beards, mustache, glasses, and sunglasses create an enormous deal of challenge. Sunglasses in particular make it difficult as they are commonly used during driving and not only they cover a significant area of the face but also cause a shadow on it which interprets many of the existing detection technologies are ineffective. Hence regardless of significant research in this region, today only a few expensive yawning monitoring systems subsist.

The following sections will describe implemented system in detail.

\section{HEAD MOVEMENT DETECTION}

This section includes software implementation. The first step towards head movement detection is detection of the driver's face. In this system the day - night camera is placed in front of driver's seat on dashboard. That camera keeps continuous watch on driver's behavior and gestures and catches continuous images in video feed. This video feed is divided into number of frames. On each frame following steps are performed.

\section{A. Blurring}

Digital cameras often have some noise in pictures. Hence to reduce noise in images blurring is applied. Before blurring RGB splitting is applied as input image is in RGB format. A 
$5 \mathrm{X} 5$ matrix is considered to get the value of surrounding pixel as follows :

$$
\left|\begin{array}{lllll}
0 & 0 & 0 & 0 & 0 \\
0 & 0 & 0 & 0 & 0 \\
0 & 0 & 1 & 0 & 0 \\
0 & 0 & 0 & 0 & 0 \\
0 & 0 & 0 & 0 & 0
\end{array}\right|
$$

Middle pixel (24bit) is located and individual pixel color value (24-bit) are read. The (8bit) separate values of R,G,B for middle pixel are calculated by using splitting technique.

$\mathrm{B}=$ pix \& 0xff

$\mathrm{G}=($ pix $>8) \& 0 \mathrm{xff}$

$\mathrm{R}=($ pix $\gg 16) \& 0 x f f$

(1), (2), (3) formulae are responsible for splitting of R (8bit), $\mathrm{G}$ (8bit), B (8bit) values of a pixel. After splitting sum of R,G,B values are calculated recursively (4), (5),(6).

$$
\begin{aligned}
& \operatorname{sumR}=\operatorname{sum} R+R \\
& \operatorname{sum} B=\operatorname{sum} B+B \\
& \text { sumG }=\operatorname{sum} G+G
\end{aligned}
$$

After that average value of middle and surrounding 24 pixels is calculated (7), (8), (9).

$$
\begin{aligned}
& \mathrm{R}=\operatorname{sumR} / 25 ; \\
& \mathrm{G}=\operatorname{sum} \mathrm{G} / 25 ; \\
& \mathrm{B}=\operatorname{sumB} / 25 ;
\end{aligned}
$$
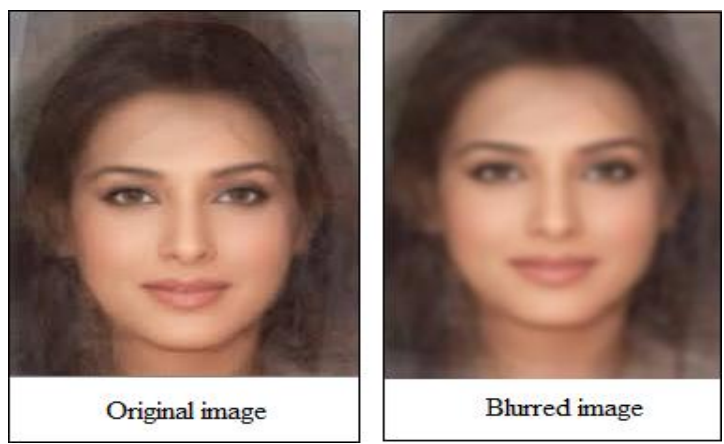

Figure 3. Blurring

Then the average value of all pixels is assigned to middle pixel.

$$
\left|\begin{array}{lllll}
0 & 0 & 0 & 0 & 0 \\
0 & 0 & 0 & 0 & 0 \\
0 & 0 & 0 & 0 & 0 \\
0 & 0 & 0 & 0 & 0 \\
0 & 0 & 0 & 0 & 0
\end{array}\right|
$$

Figure 3 shows the result of blurring.

\section{B. RGB to HSV conversion}

HSV offers more intuitive representation of relationship between colors than that of RGB. HSV select more specific colors. In HSV model value of ' $\mathrm{H}$ ' and 'S' remains constant if the value of ' $V$ ' changes.
After blurring minimum value and maximum values from $\mathrm{R}, \mathrm{G}, \mathrm{B}$ are calculated and maximum value is assigned to ' $\mathrm{V}$ '.

If $\mathrm{V}=0$ then output color is black i.e. there is no brightness in the color hence $\mathrm{H}$ and $\mathrm{S}$ will be zero.

If $\mathrm{V}$ equal to some number then $\mathrm{S}$ is calculated

$\mathrm{S}=255 *($ Max-Min $) /$ value

If we get $\mathrm{S}=0$, assign $\mathrm{H}=0$.

Now according to maximum value from R,G,B formulae (11), (12), (13) are applied to calculate $H$ respectively :

$$
\begin{aligned}
& \mathrm{H}=0+43 *(\mathrm{G}-\mathrm{B}) /(\text { Max-Min }) \\
& \mathrm{H}=85+43 *(\mathrm{~B}-\mathrm{R}) /(\text { Max-Min }) \\
& \mathrm{H}=171+43 *(\mathrm{~B}-\mathrm{R}) /(\text { Max-Min })
\end{aligned}
$$
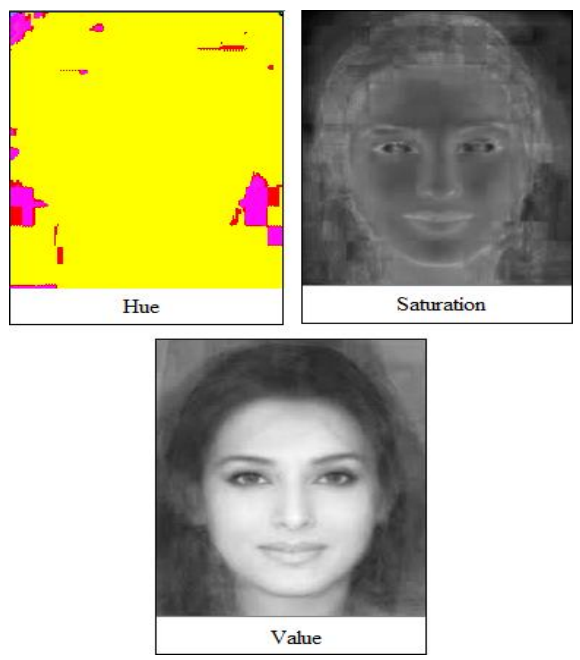

Figure 4. HSV image

C. HSV Thresholding

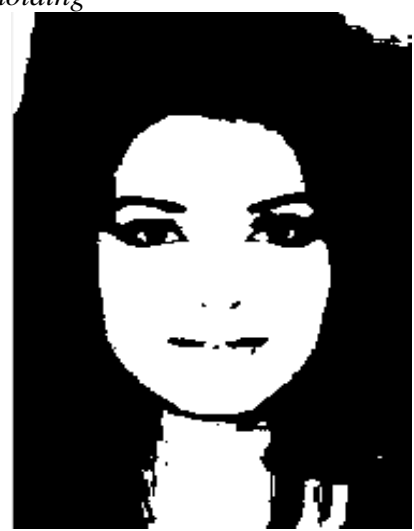

Figure 5. An isolated image using HSV Thresholding

Thresholding in HSV is very useful for isolating image features. From a grayscale image, thresholding is used to create binary image i.e. image with only black or white color. In order to isolate driver's face skin color is given as threshold value. Each 24-bit pixel value is converted into grayscale.(14)

$$
\mathrm{GS}=(\mathrm{R}+\mathrm{G}+\mathrm{B}) / 3
$$

After this binary output pixel value is calculated based on current threshold i.e. skin color as shown in Figure 5.

\section{Blob Detection}


Blob Detection mainly aimed at detecting region that differs in properties such as brightness or color as compared to surrounding. The advantage of blob detection is to provide complementary information about regions, which is not obtained from edge detectors or corner detectors. In the proposed system driver's face differs from surrounding as a result of HSV thresholding. Hence it is detected as a separate blob.

If pixel count is greater than certain limit we can consider it as a blob to be processed further.

After blob detection offset is calculated .The offset value is the distance between initial position (15) and new head position. If head position changes beyond certain limit, offset varies accordingly (16). Hence when offset value changes alert is provided .

$\mathrm{CY}=\mathrm{ySum} /$ totalPixels

Offset $=$ CY - set $Y$

\section{YAWNING DETECTION}

Yawning detection is carried out with hardware implementation. IR sensors are used for yawning detection. The sensor is placed close to driver's face. If driver yawns, sensor value changes instantly. The system basically consists of day-night camera, microcontroller, vibrator, LEDs, ADC, device driver, relay. Microcontroller i.e. AT89c51 is used to perform various functions and operations. Sensors are connected to ADC and further ADC is connected to microcontroller. The change in sensor value is detected and if greater than threshold value, signal is transferred to device driver and yawn is detected. RS-232 is used for serial communication. Figure 6.

\section{EXPERIMENTAL RESULT}

J2SE platform is used to develop the code. An external library Jmyron is used for interfacing implemented code with the camera. The system is tested by interfacing web camera to PC. Once system is initialized, camera starts capturing video feed. It grabs 10 frames per second which meets the need of real time lethargy detection. The input is taken in $320 \times 240$ format in RGB color model. After initialization it allows to select threshold and offset value manually according to driver's face and head position respectively. The offset value can be set from 10 to $20 \mathrm{~cm}$. Initial head position is set according to driver's head position. After selecting position, image processing is applied on each frame grabbed by camera successively and head position is tracked. If head movement is detected, offset changes accordingly. It indicates driver is lethargic and alert is provided. In the proposed system vibrators which are operated on $12 \mathrm{~V}$ and text to speech conversion are used as an alert mechanism. For yawning detection a sensor is placed near to driver's mouth. The optimal distance is $6-7 \mathrm{~cm}$ from driver's face. The initial value of sensor is 10-15. If driver yawns sensor value changes upto 3545 instantly which indicates the driver is lethargic and alert is provided.

\section{FUTURE SCOPE}

1. Real time detected data of driver's lethargy can be stored on the cloud system to monitor driver's performance.

2. LAB/Edge Detection Algorithm can be used for Face Detection.

3. Rather using alarm Automatic Braking System can be used which will reduce the speed of the vehicle.

4. The vehicle can be automatically parked by first using Automatic braking system, which will reduce the speed and concurrently will turn on the parking lights of the vehicles

5. Using Pressure sensor on the steering alarm Automatic braking System can be set in case of drowsiness.

7. By using wireless Technology if the driver gets drowsy an alert message can be sent to a selected person's mobile by using GSM module along with the alarm in vehicle.

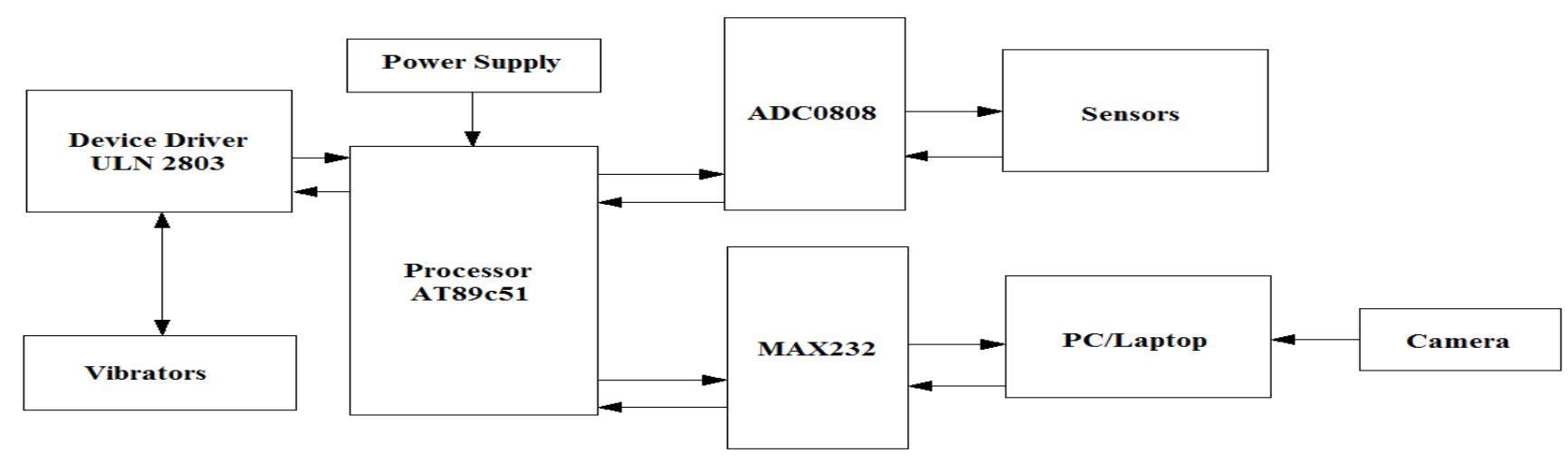

Figure 6. Block Diagram for Lethargy Detection and Alert Mechanism

\section{REFERENCES}

[1] Figure [1] IJCA Archieves Volume 132 - Number 5 http://www.ijcaonline.org/reserach/volume132/number5 dharmadhikari-2015-ijca-907349.pdf

[2] S.G. Klauer, T. A. Dingus, Neale , V. L. , Sudweeks , J.D. , and Ramsey, DJ, "The Impact of Driver Inattention on Near- Crash/Crash Risk: An Analysis Using the 100Car Naturalistic Driving Study Data," Virginia Tech
Transportation Institute, Technical Report \# DOT HS 810594.

[3] W. Qiong, Y. Jingyu, R. Mingwu and Z. Yujie, "Driver Fatigue Detection: A Survey," The Sixth World Congress on Intelligent Control and Automation, vol. 2, pp. 85878591,2006

[4] Tuner, L. ven , F. Co kun and E. Karsl gil, Vision based lane keeping assistance control triggered by a driver inattention monitor," in IEEE International Conference 
on Systems Man and Cybernetics (SMC), Istanbul, 10-13 Oct. 2010

[5] T. Pilutti and A. Ulsoy, "Identification of driver state for lane-keeping tasks," IEEE Transactions on Systems, Man and Cybernetics, Part A: Systems and Humans, vol. 29, no. 5, pp. 486-502, 1999.

[6] A. Picot, S. Charbonnier and A. Caplier, "On-Line Detection of Drowsiness Using Brain and Visual Information," IEEE Transactions on Systems, Man and Cybernetics, Part A: Systems and Humans, no. 99, pp. 112, 2011.

[7] G. Furman, A. Baharav, C. Cahan and S. Akselrod, "Early detection of falling asleep at the wheel: A Heart Rate Variability approach," Computers in Cardiology, pp. 1109-1112, 2008.

[8] S. Hu and R. Bowlds, "Pulse wave sensor for nonintrusive driver's drowsiness detection," in Engineering in Medicine and Biology Society, 2009. EMBC 2009. Annual International Conference of the IEEE, Minneapolis, MN, 2009.

[9] K. Hayashi, K. Ishihara, H. Hashimoto and K. Oguri, "Individualized drowsiness detection during driving by pulse wave analysis with neural network," in Proceedings of the 8th International IEEE Conference on Intelligent Transportation Systems, Austria , 2005.

[10] S. Sirohey, "Human Face Segmentation and Identification," in Technical Report CS-TR-3176, Maryland, 1993.

[11] H. Graf, T. Chen, E. Petajan and E. Cosatto, "Locating Faces and Facial Parts," in Proc. First Int'l Workshop Automatic Face and Gesture Recogniction, 1995.

[12] C. Han, H. Liao, K. Yu and L. Chen, "Fast Face Detection via Morphology-Based Pre-Processing," in Proc. Ninth Int'l Conf. Image Analysis and Processing, 1998.

[13] Y. Ying, S. Jing and Z. Wei, "The Monitoring Method of Driver's Fatigue Based on neural network," in International Conference on Mechatronics and Automation, 2007, Harbin, 2007.

[14] D. Saxe and R. Foulds, "Towards Robust Skin Identification in Video Images," in Proc. Second Int'l Conf. Automatic Face and Gesture Recognition, 1996.

[15] I. Craw, H. Ellis and J. Lishman, "Automatic Extraction of Face Features," Pattern Recognition Letters, vol. 5, pp. 183-187, 1987.

[16] X. Liu, G. Geng and X. Wang, "Automatically face detection based on BP neural network and Bayesian decision," in Sixth International Conference on Natural Computation (ICNC), Yantai, Shandong, 2010.

[17] X. Fan, B. Yin, Y. Fun. "Yawning Detection For Monitoring Driver Fatigue.” In: Proc. Sixth International
International Journal of Computer Applications (0975 - 8887)

Volume 141 - No.12, May 2016

Conf. on Machine Learning and Cybernetics, Hong Kong, 2007, pp. 664-668.

[18] T. Azim, M.A. Jaffar, A.M. Mirza. "Automatic Fatigue Detection of Drivers through Pupil Detection and Yawning Analysis." In: Proc. Fourth International Conf. on Innovative Computing, Information and Control, 2009, pp. 441-445.

[19] T.Wang, P. Shi. "Yawning Detection For Determining Driver Drowsiness.” IEEE International Workshop VLSI Design \& Video Tech. Suzhou

[20] M. Saradadevi and P. Bajaj, "Driver Fatigue Detection Using Mouth and Yawning Analysis," IJCSNS International Journal of Computer Science and Network Security, vol. 8, no. 6, pp. 183-188, June 2008.

[21] E. Vural, M. Cetin, A. Ercil, G. Littlewort , M. Barlett and J. Movellan, "Drowsy Driver Detection Through Facial Movement Analysis".

[22] M. Sigari, "Driver Hypo-vigilance Detection Based on Eyelid Behavior," in Seventh International Conference on Advances in Pattern Recognition, 2009.

[23] D. Liu, P. Sun, Y. Xiao and Y. Yin, "Drowsiness Detection Based on Eyelid Movement," in 2010 Second International Workshop on Education Technology and Computer Science (ETCS), 2010.

[24] M. Omidyeganeh, A. Javadtalab and S. Shirmohammadi, "Intelligent driver drowsiness detection through fusion of yawning and eye closure," in IEEE International Conference on Virtual Environments Human-Computer Interfaces and Measurement Systems (VECIMS), 2011.

[25] P. Viola and M. Jones, "Robust real-time face detection," International Journal of Computer Vision, vol. 57, no. 2, pp. 137-154, 2001.

[26] L. Li, Y. Chen , Z. Li. "Yawning Detection for Monitoring Driver Fatigue Based on Two Cameras." In: Proc. 12th International IEEE Conf. on Intelligent Transportation Systems, St. Louis, MO, USA, 2009, pp. 12-17.

[27] L. Li, Z. Li and Y. Chen, "Yawning detection for monitoring driver fatigue based on two cameras," in 12th International IEEE Conference on Intelligent Transportation Systems, 2009.

[28] X. Fan, B. Yin and Y. Sun, "Yawning Detection for Monitoring Driver Fatigue," in International Conference on Machine Learning and Cybernetics, 2007.

[29] R. Jimenez, F. Prieto and V. Grisales, "Detection of the Tiredness Level of Drivers Using Machine Vision Techniques", Electronics, Robotics and Automotive Mechanics Conference, 2011.

[30] H. Selleahewa, S. Jassim, "Image-Quality-Based Adaptive Face Recognition ", IEEE Transaction on vol 59 , no.4. 